\title{
Agro-Relatos. Periódico Universitario para el desarrollo de competencias de escritura y pensamiento crítico en estudiantes de Ciencias Agrarias
}

Luz Elena Santacoloma Varón*

DOI: https://doi.org/10.33571/revistaluciernaga.v12n23a9

\section{Resumen}

Se evalúa el impacto del periódico universitario "Agro- Relatos", estrategia generada para desarrollar las competencias de escritura y pensamiento crítico de estudiantes de Agronomía, Ingeniería agroforestal y Zootecnia. Para ello se seleccionó, una muestra representativa de 50 trabajos, en tres cohortes consecutivas de estudiantes. A estos se les aplicó una rúbrica, relacionada con las categorías: habilidades en escritura y pensamiento crítico. Los resultados se consolidaron en tablas de frecuencia por rangos de 20 puntos, de acuerdo al cumplimiento de cada cualidad y en el análisis se pudo constatar que la motivación que suscita en los estudiantes la publicación de sus narrativas en el periódico virtual tuvo impacto favorable en las competencias evaluadas.

Palabras clave: Educción, periódicos; escritura; pensamiento crítico; competencias.

Recibido. Abril 01, $2019 \quad$ Aceptado. Mayo 07, 2019

*Zootecnista, Magister en Gestión ambiental para el Desarrollo Sostenible, Doctora en Desarrollo Sostenible, Docente asociada de la Universidad Nacional Abierta y a Distancia-UNAD. Bogotá, Cundinamarca, Colombia, correo electrónico; orcid: https://orcid.org/0000-0003-2731-6825 ; e-mail: luz.santacoloma@unad.edu.co 


\title{
Agro-narratives. University newspaper for the development of writing skills and critical thinking in students of Agricultural Sciences
}

Luz Elena Santacoloma Varón*

DOI: https://doi.org/10.33571/revistaluciernaga.v12n23a9

\begin{abstract}
The impact of the university newspaper "Agro- Relatos" is evaluated, a strategy generated to develop the writing and critical thinking skills of Agronomy students, Agro-Forestry Engineering and Zootechnics. For this purpose, a representative sample of 50 works was selected, in three consecutive student cohorts. A rubric related to the components: writing skills and critical was used. The results were compiled in frequency tables by ranges of 20 points, according to the accomplishment of each quality. Through the analysis, it was possible to confirm that the publication of the narratives on the virtual newspaper was a high motivation for students and impacted positively on the evaluated competencies.
\end{abstract}

Keywords: Education, newspapers; writing; critical thinking; competencies.

Received. April 01, 2019

Accepted. May 07, 2019

\footnotetext{
* Zootechnician, Master in Environmental Management for Sustainable Development, Doctor in Sustainable Development, Associate Professor at Universidad Nacional Abierta y a Distancia-UNAD. Bogotá, Cundinamarca, Colombia, e-mail; orcid: https://orcid.org/0000-0003-2731-6825 ;e-mail: luz.santacoloma@unad.edu.co
} 


\section{Agro-Estórias. Jornal universitário para o desenvolvimento de habilidades de escrita e pensamento crítico em estudantes de Ciências Agrárias}

Luz Elena Santacoloma Varón*

DOI: https://doi.org/10.33571/revistaluciernaga.v12n23a9

\section{Resumo}

É avaliado o impacto do jornal universitário «Agro- Relatos», estratégia gerada para desenvolver a capacidade de escrita e pensamento crítico dos estudantes de Agronomia, Engenharia AgroFlorestal e Zootecnia. Para este fim, uma amostra representativa de 50 trabalhos foi selecionada em três coortes consecutivas de estudantes. Uma rubrica foi aplicada a estes, relacionada com as categorias: habilidades de escrita e pensamento crítico. Os resultados foram consolidados em tabelas de freqüência por faixas de 20 pontos, de acordo com o cumprimento de cada qualidade $e$ na análise foi possível verificar que a motivação que os estudantes têm para publicar suas narrativas no jornal virtual teve um impacto favorável sobre as competências avaliadas.

Palavras chave: Educação, jornais; escrita; pensamento crítico; competências.

Recebido.Abril 01, $2019 \quad$ Aceitado. Maio 07, 2019

\footnotetext{
* Zootecnista, Mestre em Gestão Ambiental para o Desenvolvimento Sustentável, Doutorada em Desenvolvimento Sustentável, Professor Associado na Universidade Nacional Aberta e na Distancia-UNAD. Bogotá, Cundinamarca, Colômbia, e-mail; orcid: https://orcid.org/0000-0003-2731-6825 ;
} e-mail: luz.santacoloma@unad.edu.co 


\section{Introducción}

Los estudiantes del curso de Política agraria y ambiental de la UNAD pertenecen a los programas de Agronomía, Zootecnia e Ingeniería Agroforestal; geográficamente están ubicados en todo el territorio nacional, en coherencia con el modelo de educación Abierta y a Distancia que implementa esta universidad. Su carácter de estudiantes de ciencias agrarias les otorga una gran sensibilidad frente a las problemáticas agrarias de sus regiones, particularmente agudas en Colombia, como consecuencia de innumerables conflictos históricos que aún perviven en los territorios rurales. Por tanto, dentro del curso de Política Agraria y Ambiental se abre un espacio para la reflexión, sobre estas temáticas, en la cual los estudiantes a través de crónicas, entrevistas, reportajes e historietas interpretan sus realidades, deliberan acerca de conflictos que identifican en sus regiones y estas narrativas, al ser seleccionada por su importancia, profundidad y adecuada redacción, se hace pública a través del e-periódico denominado "Agro - Relatos".

Lo anterior se enmarca dentro del concepto "“pensar en público", que defiende Hoyos (2011), como condición para la construcción de democracia y ciudadanía; para este autor, hacer públicas las ideas, permite tejer una red de intereses comunes, como resultado de la tolerancia, "virtud y forma de lo público", que surge a partir de la confrontación de ideas y de diferencias y desacuerdos, también señala que "la educación es comunicación y ésta es la competencia ciudadana por excelencia". Estas reflexiones permiten inferir que al hacer públicas las ideas, es posible la construcción de tejido social, interacción e interconexión, y ello es coherente, con lo expuesto por Alcázar y Villamizar (2006), cuando afirman que las organizaciones modernas, llámense públicas o privadas, deben socializar sus acciones, lo que equivale a volverlas públicas.

Navarro, (2010) afirma que, un medio que permita publicar diversidad de posiciones, de ideologías, de tendencias políticas, genera vínculos de solidaridad, sentido colectivo y confianza entre los actores sociales; sus mensajes movilizan comunidades probablemente silenciadas, restablecen la interacción y recuperan la posibilidad de lo público. Este autor defiende la existencia de medios, que permitan:

"reconstruir lo público, proponer nuevas formas de vida, ciudadanía, identidades, demandas, nuevas formas de sentir y de pensar, expresar, narrar, nuevas estéticas; en pocas palabras, nuevas formas de comunicar".

Para Zemelman (2004), la formación de ciudadanía se expresa como la capacidad de deliberar y reflexionar con el otro; manifiesta que la socialización política requiere paradigmas que rompan el miedo, la apatía, la incredulidad y el escepticismo, lo cual se obtiene potenciando la capacidad de pensar críticamente, superar los límites de la vida privada al encontrar sentido en los escenarios públicos, en los que pueda jugar la pluralidad como acción y como narrativa. Lo anterior lo resume (Cubides, 2004) en lo siguiente; "se trata de recuperar su capacidad de pensar por sí mismo, sin desconocer al otro, y reconocer, crear, disponer en la práctica los principios que orientan la vida".

En este sentido, la estrategia comunicativa del periódico "Agro - Relatos" alude al ideal Kantiano, de un sujeto que se atreve a pensar por sí mismo, que muestra su mayoría de edad mediante el análisis, reflexión de las situaciones que lo rodean y lo expresa de manera pública, en relación dialógica. Sobre el particular, Cubides (2004), expone que la autonomía, la reflexividad y la conciencia histórica tienen que ver con la posibilidad de decidir y actuar en los contextos sociales, con quienes se interactúa. 
Además de lo expuesto, el espacio, académico del e-periódico, abre horizontes de conocimiento al estudiante, con la lectura e interpretación de problemáticas regionales, y el análisis de sus posibles soluciones, dentro de un ámbito estético, que implique proyectar sobre las situaciones encontradas una mirada singular, con libertad de expresión y de lenguajes. Sánchez (2015), considera que las actividades que estimulan la creatividad son un factor de motivación para procesos de aprendizaje, y cambios actitudinales que incentiven la perseverancia y la dedicación; por lo cual, es necesario fomentar en los estudiantes, actividades gratificantes, que estimulen la habilidad de pensar de forma crítica.

Por su parte, la utilización de tecnologías de Información y comunicación en procesos de aprendizaje, en los cuales se promueve la autonomía y la responsabilidad, no son herramientas simples, ya que pueden constituir nuevas formas de conversación, estéticas, narrativas, vínculos relacionales, modalidades de construcción de identidades y de perspectivas sobre el mundo (Lugo, 2008).

A lo anterior, se añade que los estudiantes en el mundo contemporáneo deben adicionar a las habilidades clásicas relacionadas con lectura, escritura y matemáticas, competencias que les permita sentirse cómodos con la colaboración, la formulación de preguntas, el desarrollo de pensamiento autónomo y crítico, teniendo presente la ciudadanía responsable (Buxarrais, 2016). En este paradigma educativo, se privilegia a una comunidad que busca, selecciona, construye y comunica conocimiento colaborativamente en un tipo de experiencia que se conecta directamente con el concepto de comunidades de aprendizaje (Organización de Naciones Unidas, 2013).

En las comunidades de aprendizaje que se constituyen mediante el uso de estrategias como el e-periódico "Agro -Relatos", uno de los principales objetivos es la generación de pensamiento crítico, definido según Causado, (2015) como el arte de discernir y establecer una posición a partir de los argumentos, en la que se logra independencia y emancipación. Al respecto, Guzmán y Sánchez, (2006), expresan que se hace necesaria la implementación de prácticas explícitas que permitan la adquisición de estas competencias ya que si bien, el conocimiento es esencial para generar pensamiento, este no garantiza el desarrollo del pensamiento crítico. No obstante, Ossa, Palma, Lagos, Quintana y Díaz (2017) señalan, que es muy difícil realizar una evaluación de pensamiento crítico, dado que existen una gran variedad instrumentos y de conceptos, que no han permitido un consenso acerca de su verdadero sentido.

El pensamiento crítico conduce al dominio de contenidos, al aprendizaje profundo y exige la defensa de posiciones frente a temáticas complejas, al contrastarla con teorías y puntos de vista de diferentes autores. Esta forma de pensamiento también demanda la evaluación de hechos, sus implicaciones y consecuencias, y la aceptación de contradicciones e inconsistencias de su propio pensamiento y experiencia (Paul \& Elder, 2005). Desde diferentes perspectivas, se coincide en que para promover el pensamiento crítico se debe prestar atención al desarrollo de las creencias epistemológicas de los estudiantes, facilitar un aprendizaje activo alrededor de problemas relacionados con situaciones de la vida real y estimular interacciones entre los estudiantes que posibiliten hablar y compartir distintas formas de ver el mundo (Oliveras \& Sanmartí 2009).

Los autores citados, coinciden en que el pensamiento crítico incluye enunciar hipótesis, analizar problemas desde diferentes puntos de vista, plantear nuevas preguntas y posibles soluciones, y planificar estrategias para investigar. Betancourth, (2015), explica que el desarrollo de habilidades de pensamiento crítico también incluye la capacidad de seleccionar muy bien las fuentes de información, valorar la calidad de los argumentos y elaborar un punto de vista propio sobre asuntos problemáticos de la vida cotidiana. 
Por tanto, es importante estimular formas de pensamiento de orden superior, que para Tung y Chang (2009), son un proceso cognitivo complejo que busca ante todo evaluar, procesar en forma analítica y aceptar o rechazar información socialmente construida, y para López (2012), incide en rasgos como la apertura mental y la forma en las cuales se enfrentan los estudiantes a los retos de la vida.

\section{METODOLOGÍA}

\subsection{Primera Fase.}

\section{- Selección del curso}

Para estimular la generación de competencias de escritura y pensamiento crítico, se seleccionó el curso de Política Agraria y Ambiental, ya que, se estudian temáticas, relacionadas con el histórico conflicto agrario que aún persiste en Colombia y que cobra relevancia en la actualidad, debido a que el tema agrario está nuevamente en las agendas públicas de los territorios. De otra parte, la presencia de diferentes tipos de conflicto dependiendo de la región donde están ubicados los estudiantes de los programas de Agronomía, Ingeniería Agroforestal y Zootecnia, de la UNAD, enriquece el conocimiento y análisis de estas situaciones desde una perspectiva crítica. Se parte de la premisa de que los estudiantes al ser motivados con la publicación de su relato en el e-periódico, mejora, las competencias escritoras y motiva el desarrollo de pensamiento crítico.

Es importante señalar que autores como Saiz, \& Rivas, (2008), expresan que las capacidades intelectuales por sí mismas, no consiguen la eficacia que se asume en pensamiento crítico, sino que es necesario la motivación, la cual pone en funcionamiento estas capacidades. Al respecto, Ennis (2008), uno de los autores que más ha trabajado en evaluación de pensamiento crítico, plantea que la mayoría de las pruebas fracasan debido a que suelen ser exclusivamente objetivas, razón por lo cual, la rúbrica elaborada para el presente análisis contempla aspectos cualitativos de gran relevancia en la formación de pensamiento crítico.

\section{- Descripción de la actividad}

En una de las tareas del curso de Política Agraria y Ambiental seleccionado en el presente estudio, cada estudiante identificó una problemática agraria en su región, desarrolló un proceso investigativo, el cual incluyó trabajo de campo, entrevistas, documentos, recolección de datos, entrevistas, entre otros. Estos documentos y la narración final acerca del problema identificado, se compartió en un foro de trabajo colaborativo con sus compañeros (cinco integrantes), quienes realizaron aportes pertinentes a cada uno de ellos. Cada estudiante tuvo la posibilidad de revisar los escritos de sus compañeros y dar sus sugerencias y percepciones sobre el trabajo, y a partir de estas observaciones, se realizaron ajustes, considerando la retroalimentación del equipo de trabajo colaborativo.

El resultado final fue una crónica, entrevista, documental o reportaje, con los elementos propios de cada género, que recibió los aportes del grupo. Esta dinámica permitió incorporar en el proceso aprendizajes cooperativos los cuales fomentaron habilidades de orden superior, como análisis, disertación crítica y valoración. Los docentes responsables del curso realizaron una selección de los relatos, que presentaron mayores puntajes para los cuales se tuvo en cuenta la rúbrica elaborada con los elementos expuestos en la Tabla 1, y estos fueron publicados en e-periódico "Agro - Relatos", el cual es de libre circulación y constituye un material de consulta para los estudiantes de los programas de agronomía, Ingeniería Agroforestal y Zootecnia. 
En la Figura 1, se aprecia el esquema seguido para la actividad del e-periódico, con el uso de Tecnología de Información y Comunicación (TIC), y con la cual se motivaron las competencias de escritura y pensamiento crítico. En la figura se advierte que la estrategia estuvo atravesada por dos ejes conceptuales, que son la cultura política y el pensar en público mediante la interpretación de los contextos regionales.

Figura 1. Esquema de la actividad e- periódico para estimular competencias de escritura y pensamiento crítico

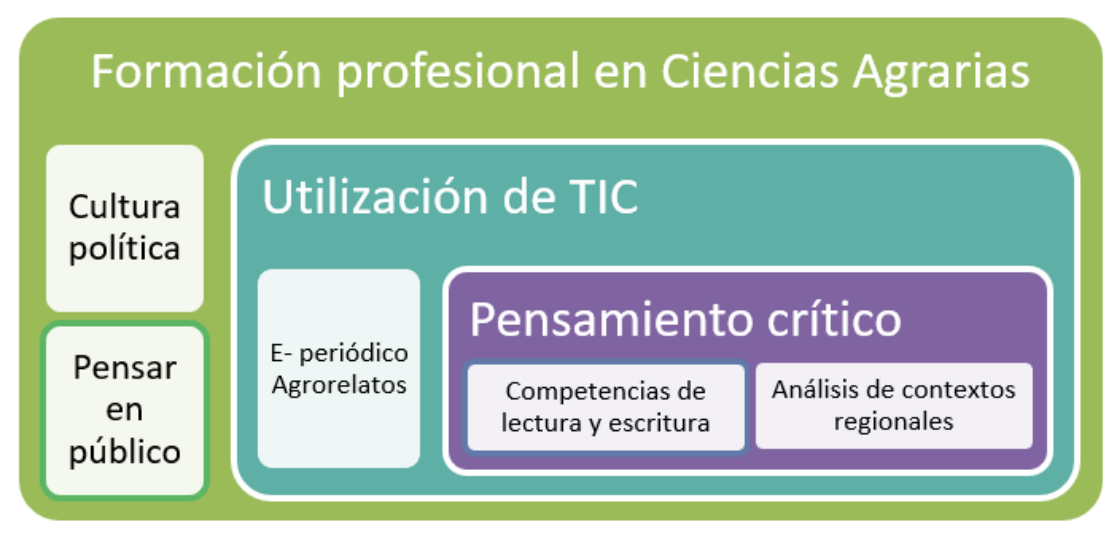

Fuente Propia

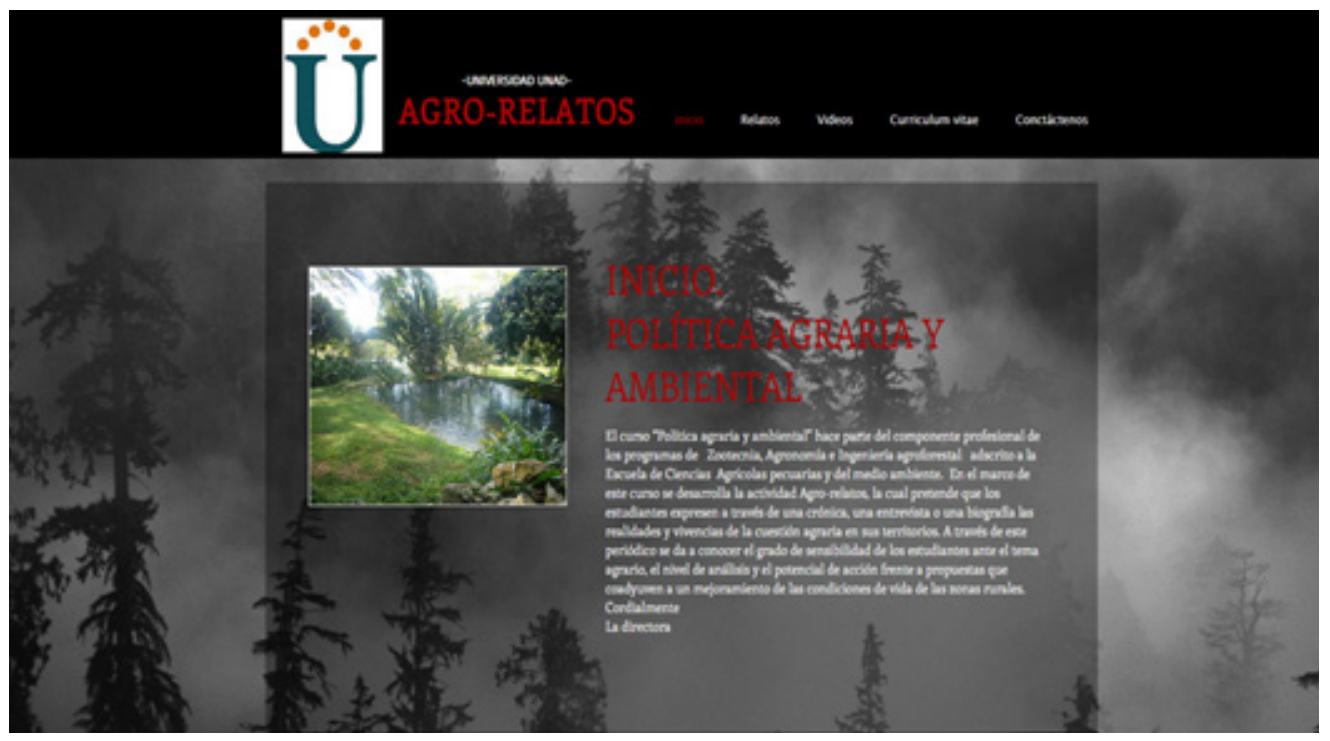




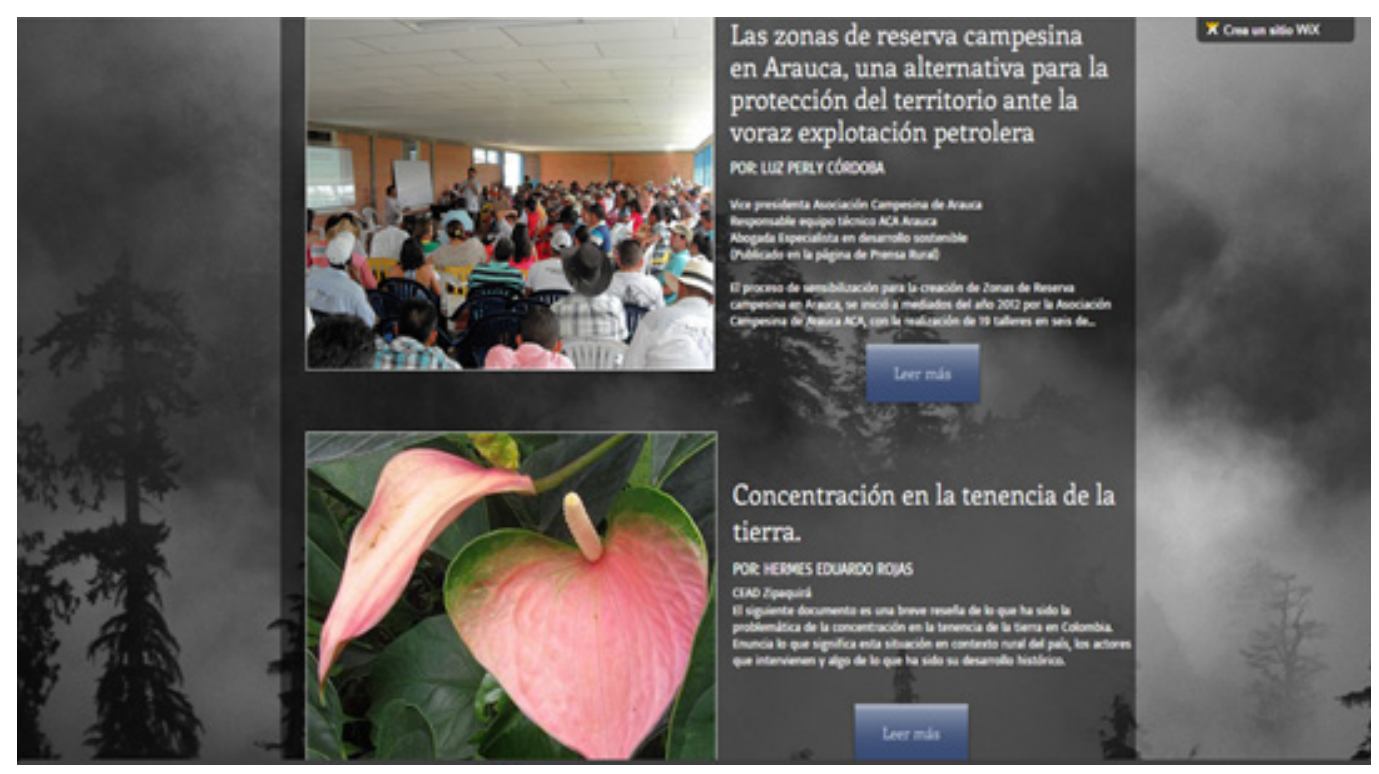

\subsection{Segunda fase}

\section{- Población objetivo}

El estudio se desarrolló al establecer una comparación del desempeño en competencias de escritura y pensamiento crítico de los estudiantes entre un grupo control (segundo período de 2015) y el de dos cohortes sucesivas (primer período de 2016 y segundo período de 2016). Los estudiantes de estas dos últimas cohortes tuvieron la motivación de que sus trabajos podrían ser publicado en el e- periódico "Agro - Relatos". Con el propósito de establecer la comparación, en cada cohorte fueron seleccionados por muestreo aleatorio simple cincuenta (50) relatos presentados por los estudiantes de Agronomía, Ingeniería Agroforestal y Zootecnia, los cuales constituyeron una muestra representativa con un nivel de confianza (1- $\alpha$ ), de una población $\mathrm{N}=400$ estudiantes en cada una de las cohortes, asumida como universo o población.

Cada trabajo seleccionado, se constituyó como unidad de análisis, con la aplicación de una rúbrica evaluativa para las categorías de escritura y pensamiento crítico. Cada una de estas categorías se conformó de diversas cualidades, las cuales se presentan en la Tabla 1, a las que fueron asignados puntos de acuerdo con el grado de complejidad. Los resultados se consolidaron en tablas de frecuencia, agrupando los trabajos de acuerdo con los puntajes obtenidos dentro de los siguientes rangos ( 0 a 20,21 a 40,41 a 60,61 a 80 y 81 a 100), los trabajos con mayores puntajes fueron publicados en el e-periódico. 
Tabla 1. Cualidades evaluadas en las narraciones

\begin{tabular}{|c|c|}
\hline Escritura del texto & Cualidad \\
\hline Título del relato & $\begin{array}{l}\text { Pertinencia } \\
\text { Sugestividad } \\
\text { Interrelación con el texto }\end{array}$ \\
\hline Escritura del texto & $\begin{array}{l}\text { Claridad } \\
\text { Concisión } \\
\text { Precisión } \\
\text { Coherencia } \\
\text { Orden de las ideas }\end{array}$ \\
\hline Pensamiento crítico & $\begin{array}{l}\text { Relevancia } \\
\text { Interpretación } \\
\text { Profundidad } \\
\text { Análisis } \\
\text { Argumentación } \\
\text { Inducción: generalizar a partir } \\
\text { de hallazgos } \\
\text { Conclusión }\end{array}$ \\
\hline
\end{tabular}

Para valorar las competencias de escritura se consideraron algunas cualidades, que son importantes en la comprensión de los textos presentados por los estudiantes, y en la apreciación del nivel discursivo. Al respecto, Carlino (2006), expresa que, al elaborar un texto, al compartirlo con otros, discutir sobre su redacción, es posible, objetivar mejor el nivel discursivo del estudiante. No obstante, Ortiz (2015), plantea que la escritura académica, como categoría de análisis no solo involucra conceptos y teorías sino discursos y prácticas, muchas veces difusos y otras veces firmes y hegemónicos, por lo cual es frecuente que se presenten tensiones y contradicciones en su análisis.

En la rúbrica fue asignado puntaje al título y se le otorgaron criterios de pertinencia, sugestividad (invitación a la lectura) e interrelación con el texto, en tanto que al cuerpo del relato se le asignaron criterios como; claridad, concisión, precisión, coherencia y ordenamiento de las ideas. Con respecto al pensamiento crítico se le asignaron criterios de relevancia, interpretación (del problema), profundidad en el tratamiento de la temática, análisis de la situación, argumentación, inducción y conclusión. Para definir estas categorías se tuvo presente el concepto de pensamiento crítico, dado por varios autores, como el expuesto por Saiz (2012), en el sentido de que éste dialoga con la realidad, y no se limita a profundizar en procesos argumentativos, sino en la búsqueda de soluciones a las problemáticas identificadas. 


\section{Resultados y análisis \\ 2.1 Resultados}

El objetivo de la investigación fue evaluar, hasta qué punto la publicación de las narrativas, en un medio universitario genera algún impacto en las competencias de escritura y pensamiento crítico, para lo cual se hizo un seguimiento a grupos de 50 estudiantes, durante tres períodos consecutivos, mediante la aplicación de una rúbrica de evaluación a los trabajos presentados. Los resultados de la primera cohorte, (segundo período de 2015) se pueden observar en la figura 3 , y en ellos se advierte que del $100 \%$ de los trabajos evaluados, el $80 \%$ tienen pertinencia, y $75 \%$ relevancia, esto indica que los estudiantes presentan sensibilidad frente a sus problemáticas regionales y que éstas a pesar de que son de diversa naturaleza revisten importancia para la comunidad.

Figura 3. Tablas de frecuencia que muestran el comportamiento de cada una de las cualidades evaluadas en competencias de escritura y pensamiento crítico en la primera cohorte de estudiantes (segundo período de 2015)

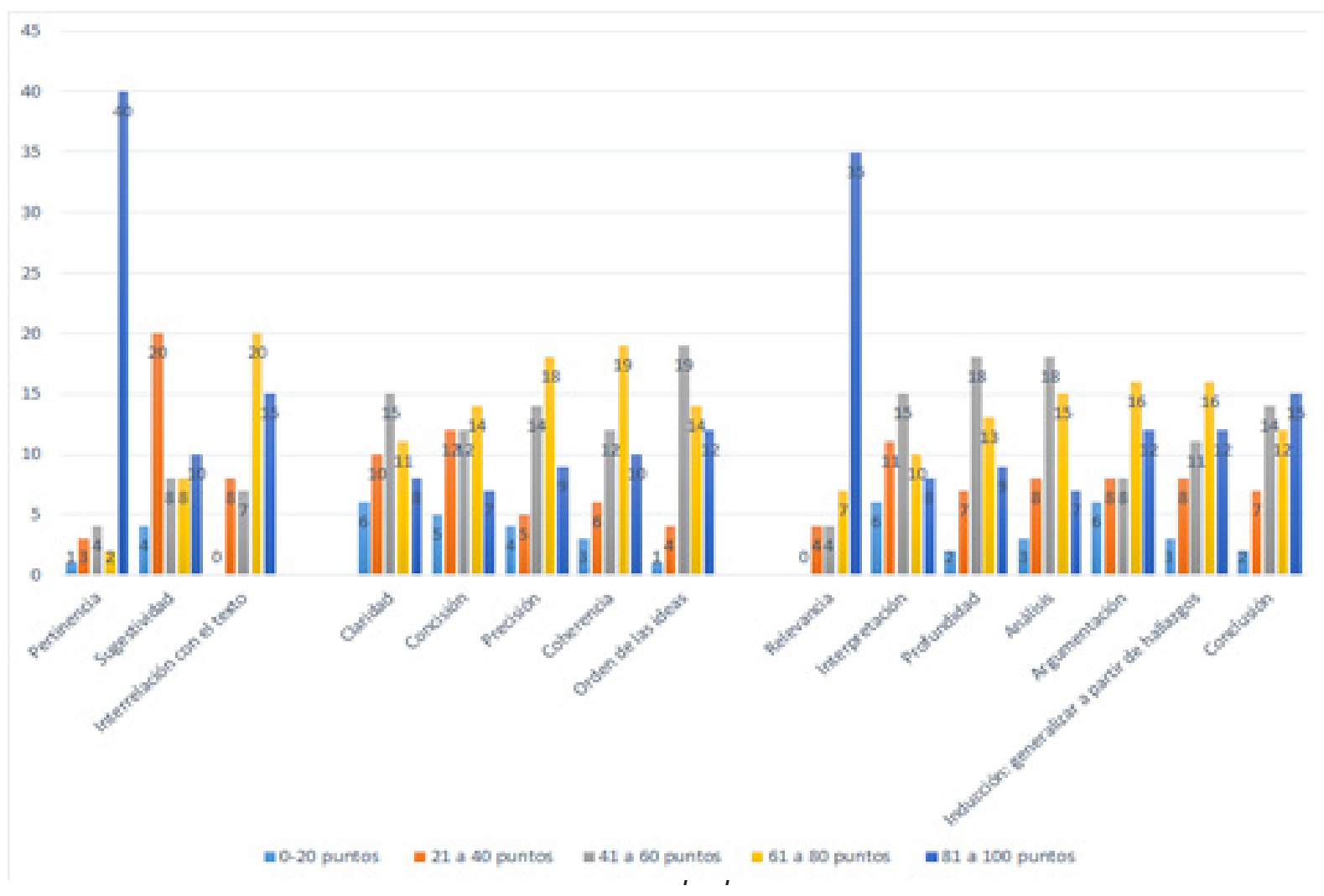

Las cualidades, interpretación, profundidad y análisis, correspondientes a la categoría de pensamiento crítico sólo el $16 \%$, el $18 \%$, y el $14 \%$ respectivamente, estuvieron en el rango de 81 y 100 puntos. 
Figura 4. Tablas de frecuencia que muestran el comportamiento de cada una de las cualidades evaluadas en competencias de escritura y pensamiento crítico en la segunda cohorte (primer período de 2016)

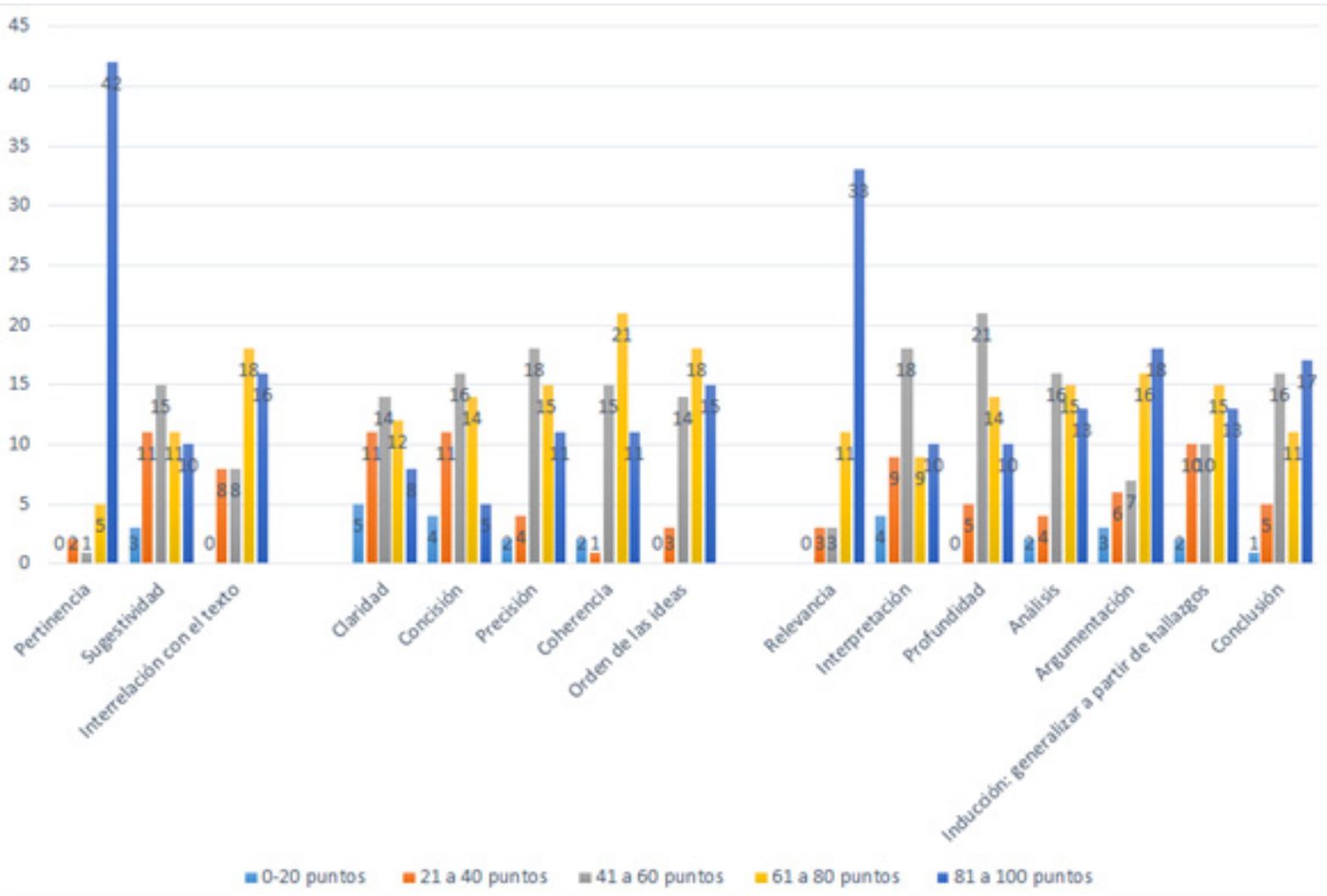

Fuente Propia. Estudio 2019

En la Figura 4, se advierte que la cualidad "orden de las ideas" correspondiente a la categoría de competencias en escritura, mejoró entre la primera y segunda cohorte ocho (8) puntos porcentuales, pasando de $28 \%$ a $36 \%$, teniendo en cuenta los estudiantes que estuvieron en el rango de 81 a 100 puntos. Por su parte, las cualidades análisis y argumentación mejoraron entre la primera y segunda cohorte en 12 puntos porcentuales los estudiantes que estuvieron en el rango de 81 a 100 puntos. Este aspecto es de destacar, si se tiene en consideración que la argumentación, constituye uno de los principales atributos para el adecuado desempeño profesional y ciudadano. 
Figura 5. Tablas de frecuencia que muestran el comportamiento de cada una de las cualidades evaluadas en competencias de escritura y pensamiento crítico en la tercera cohorte (segundo período de 2016)

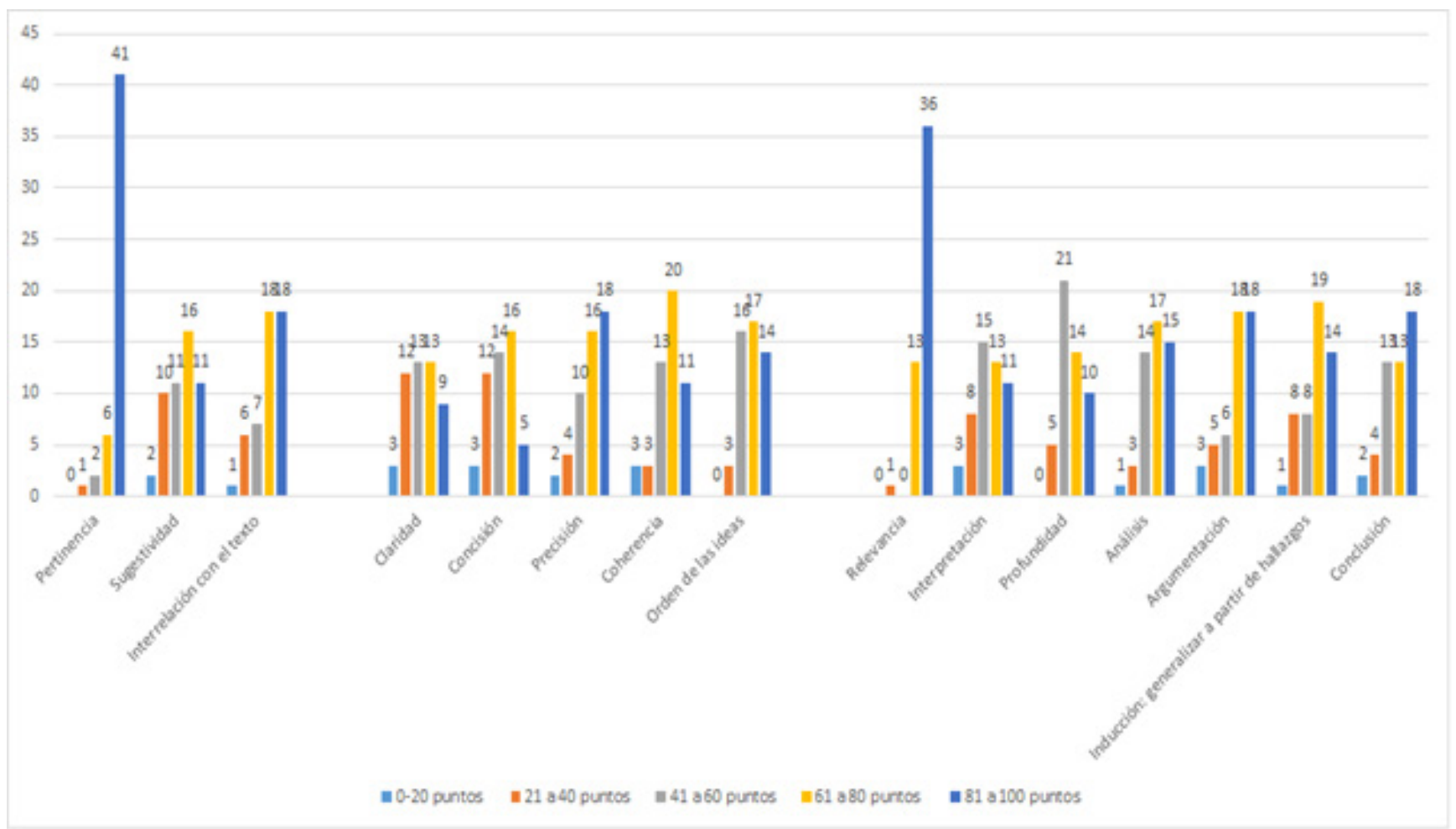

Fuente Propia. Estudio 2017

Entre la segunda y la tercera cohorte se observó un incremento de 14 puntos porcentuales, en la cualidad "precisión" correspondiente a competencias en escritura, con lo cual se aumentó de $22 \%$ a $36 \%$, los trabajos que estuvieron en los rangos de 81 a 100 puntos. De igual manera se identificó un incremento de cuatro puntos porcentuales en la cualidad "análisis" perteneciente a la categoría de pensamiento crítico, pasando de $26 \%$ a $30 \%$ el número de trabajos que estuvieron en el rango de 81 a 100 puntos. Lo anterior es atribuible a que se ha hecho mucho énfasis en que un material para publicar debe tener unas adecuadas referencias bibliográficas, ya que se determinó que uno de los puntos sensibles es el tipo de fuente utilizada. Al respecto se ha establecido que una gran parte de estudiantes hacen uso de información de la red, sin validez científica y baja confiabilidad.

Estos resultados señalan la necesidad de fomentar actividades pedagógicas que estimulen el "pensamiento" en los estudiantes e induzcan a dar gran valoración a aptitudes comprometidas con el análisis crítico de los procesos políticos y sociales en los cuales vive el estudiante

\subsection{Análisis}

Los resultados obtenidos en los trabajos de las diferentes cohortes analizadas son atribuibles a que en la mayor parte de los documentos prima la "opinión" y hay bajo grado de documentación e investigación acerca del problema identificado, del mismo modo, hay baja relación entre el problema planteado en el relato y la conceptualización teórica acerca de las políticas agrarias.

Una alta proporción de narrativas se limitan al planteamiento del problema y hay una mirada "pesimista" frente a su posible solución. Al respecto, es importante señalar que, en algunos grupos, 
de trabajo colaborativo es frecuente que los miembros del grupo den luces acerca de posibles alternativas, de tal manera que se activa el pensamiento y el resultado es una crónica con una mirada más "optimista" en la cual se tiene en cuenta la relevancia del tema agrario en la agenda política actual. Se destaca que el $38 \%$ de los documentos evaluados, obtuvieron de 61 a 80 puntos en coherencia, lo anterior muestra que esta cualidad es de alta frecuencia.

En los resultados obtenidos se identifica una dificultad en analizar las problemáticas regionales, desde la perspectiva de los aprendizajes teóricos, por lo cual, es importante adelantar esfuerzos en esta dirección, que conduzcan a que los estudiantes conecten las problemáticas con los planteamientos expuestos por autores que investigan las temáticas, con el fin de que adquieran mayor autonomía intelectual y habilidades de pensamiento.

Dado que muchos estudiantes toman como referencia información de la red, con baja validez científica, es necesario aportarles criterios de selección de información, que permita realizar un desarrollo de su trabajo con un profundo nivel de análisis, y les de elementos para plantear soluciones a los problemas identificados.

\section{Conclusiones}

De acuerdo con los resultados obtenidos con la publicación de las mejores narrativas en el e-periódico "Agro- Relatos", en el área de Política Agraria y Ambiental de estudiantes de Agronomía, Ingeniería Agroforestal y Zootecnia, se establece que las cualidades pertinencia y relevancia presentan altas frecuencias, en puntajes entre 81 a 100, lo cual revela alta sensibilidad de los estudiantes frente a problemas del entorno.

Se pudo observar que las cualidades interpretación, profundidad, y análisis, correspondientes a la categoría de pensamiento crítico, presentan el comportamiento más bajo, en las tres cohortes analizadas. La cualidad argumentación igualmente requiere un fortalecimiento a través de estrategias pedagógicas como la del periódico virtual, ya que en las diferentes cohortes obtuvo un bajo comportamiento,

El aumento entre la segunda y la tercera cohorte en la cualidad "precisión" relacionada con la competencia en escritura, y en la cualidad "análisis" perteneciente a la categoría de pensamiento crítico, indica que aquellos estudiantes que presentaron sus trabajos después de conocer el periódico virtual y de tener la motivación de publicar sus crónicas mediante este medio, obtienen mejores resultados en cualidades de escritura y pensamiento crítico.

Competencias en lectoescritura. Teniendo en cuenta que la lectura y la escritura son ejes transversales de todos los procesos académicos es difícil atribuir su mejoramiento a una actividad específica dentro de un curso académico; no obstante, la motivación que genera el "contar" y publicar una historia relacionada con el entorno del estudiante, con un problema sentido de su comunidad, anima a la producción de escritos de calidad, e induce a la realización de textos coherentes, claros y a una adecuada organización de las ideas. Se pudo concluir que la escritura y el acto de comunicar a la luz de las nuevas tecnologías permiten también el desarrollo de competencias relacionadas con la sensibilidad frente a problemas del entorno.

La publicación de narrativas por parte de miembros de la comunidad indujo igualmente a procesos de lectura por parte de la comunidad académica, dado el interés que despierta en los estudiantes la lectura de textos, elaborados por sus compañeros, y en los cuales se da a conocer problemáticas presentes en diferentes regiones del país, escritos en muchos casos por sus propios protagonistas. 
Se concluye que el estudiante de ciencias agrarias debe producir continuamente textos, redactar documentos coherentes, comprensivos, elaborados con gran sentido crítico, que permita convocar al análisis de situaciones y a generar soluciones y salidas a las problemáticas a través de la comunicación.

Competencias en pensamiento crítico. La realización de la actividad permitió que los estudiantes contribuyeran a establecer un nexo entre el mundo académico y la realidad de su entorno, y a que realizaran una lectura y comprensión de los fenómenos de sus regiones desde una perspectiva más analítica, desarrollando de esta manera habilidades de escritura y pensamiento crítico necesarias para desenvolverse en su ámbito profesional y ciudadano. Se destaca la cooperación de los miembros del grupo de trabajo colaborativo en los resultados finales de los textos, lo cual posibilitó procesos de participación y aprendizaje que los llevó a cuestionar sus puntos de vista y a discutir sus ideas iniciales.

En los relatos (crónicas, entrevistas, editoriales) seleccionados por la calidad intelectual para su publicación en el periódico "Agro - Relatos", se advierte, análisis de conceptos, defensa de posiciones en asuntos de alta complejidad, planteamiento de puntos de vista, trasferencia de ideas a nuevos conceptos y conclusiones asertivas. Lo anterior es común en los relatos seleccionados, pero en aquéllos que no fueron escogidos para su publicación, en las tres cohortes analizadas, se identifica la tendencia a la "opinión personal" con escaso nivel de fundamentación y de consulta bibliográfica válida.

De lo expuesto, se deriva que es necesario fomentar la lectura y la consulta de autores, para que los estudiantes puedan establecer conexiones entre los problemas identificados y la información teórica (resultado de investigaciones), que contribuyan dar elementos para fundamentar las opiniones. Con este tipo de actividades, también se estimula en pensamiento inductivo, el cual permite hacer generalizaciones a partir de hallazgos y problemáticas identificadas en las regiones. En este mismo sentido, es importante contribuir a que los estudiantes adquirieran criterios de selección de información, ya que, para algunos, el hecho de que esta se encuentra disponible en la red es suficiente para considerarla con validez y credibilidad.

Se concluye que la realización de la actividad, demanda un gran esfuerzo intelectual, y que ello coincide con la necesidad de que una universidad, contemporánea brinde una educación que los prepare no solo para las necesidades de hoy, sino del futuro, como es la capacidad de identificar problemas relevantes, inferencia, interpretación, argumentación, capacidad de síntesis, en un marco de investigación, de sensibilidad y compromiso frente a problemáticas de su entorno.

Para los estudiantes del curso de Política Agraria y Ambiental la publicación del periódico "Agro - Relatos", constituye una motivación, para tratar de manera analítica temas de trascendencia, como las problemáticas agrarias en los territorios de Colombia, lo cual amerita disciplina intelectual para su análisis, tratamiento adecuado de la información, y lecturas desde diferentes vertientes ideológicas. La estrategia del e-periódico, fortalece las competencias en lectura y escritura, e incita al pensamiento crítico, lo cual implica; interpretación de la realidad, contraste con diversos autores, aclaración de supuestos e interpelación de sus propios pensamientos.

Se considera que la actividad es idónea, para que el estudiante no solo interactúe con su entorno sino para que adquiera elementos para trasformar situaciones. Lo anterior es coherente con la Declaración sobre la educación superior en el siglo XXI, elaborada por la conferencia mundial de la UNESCO, que fue organizada en 1998 en París, la cual expone que "Las instituciones de educación superior deben formar a los estudiantes para que se conviertan en ciudadanos bien informados y profundamente motivados, provistos de un sentido crítico y capaces de analizar los problemas de la sociedad, buscar soluciones, aplicarlas y asumir responsabilidades sociales" 


\section{Referencias bibliográficas}

Alcázar, P. \& Villamizar, C. (2006). Barranquilla: En busca de las agendas perdidas. Revista investigación y desarrollo, 14, 372-389. Recuperado de https://www.redalyc.org/ $\underline{\mathrm{html} / 268 / 26814207 /}$

Betancourth Zambrano, S. (2015). Desarrollo del pensamiento crítico en docentes universitarios. Una mirada cualitativa. Revista Virtual Universidad Católica del Norte, 44, 238-252. Recuperado de http://revistavirtual.ucn.edu.co/index.php/RevistaUCN/article/view/627/1162

Buxarrais, M. (2016). Redes sociales y educación. Education in the knowledge 17 (2), 15-20. Recuperado de https://www.redalyc.org/pdf/5355/535554762002.pdf

Causado, R., Santos, B., Calderón, I. (2015). Desarrollo del pensamiento crítico en el área de Ciencias Naturales en una escuela de secundaria. Revista Facultad de Ciencias Universidad Nacional de Colombia, Sede Medellín, 4 (2), 17-42. Recuperado de https://revistas.unal.edu. co/index.php/rfc/article/view/51437/54884

Carlino, P (2006). Escribir, leer y aprender en la universidad. Una introducción a la alfabetización académica. Buenos Aires: Fondo de Cultura Económica, FCE

Castor Paniagua, L. (2018). Historia de los periódicos universitarios. El caso de la Universidad Autónoma de Santo Domingo-UASD. LUCIÉRNAGA, 9(18). Recuperado de https://revistas. elpoli.edu.co/index.php/luc/article/view/1261

Cubides, H. (2004). Formación del sujeto político. Escuela, medios y nuevas tecnologías de la comunicación y la información", en Laverde, M. C.; Daza, G., y Zuleta, M., Debates sobre el sujeto. Perspectivas contemporáneas, Bogotá, Universidad Central, DIUC, Siglo del Hombre Editores

Ennis, R. H. (2008). Nationwide testing of critical thinking for higher education: Vigilance required. Teaching Philosophy, 31(1), 1-26

Guzmán, S y Sánchez. P. (2006). Efectos de un programa de capacitación de profesores en el desarrollo de habilidades de pensamiento crítico en estudiantes universitarios en el Sureste de México. Revista Electrónica de Investigación Educativa. 8(2), 1-17. Recuperado de http:// www.scielo.org.mx/pdf/redie/v8n2/v8n2a2.pdf

Hoyos, G. (2011). Educación y ética para una ciudadanía Cosmopilita. 55, 191-203. Recuperado de https://www.youtube.com/watch?v=FcQoc oLjDM

López, G. (2012). Pensamiento crítico en el aula. Docencia e Investigación: revista de la Escuela Universitaria de Magisterio de Toledo, 37(22), 41-60. Recuperado de https://ruidera.uclm.es/ xmlui/handle/10578/9053

Lugo, M.T. (2008). Las políticas TIC en la educación de América Latina. Tendencias y experiencias. Revista Fuentes, 10, 52-68. Recuperado de https://idus.us.es/xmlui/handle/11441/32395

Navarro, L (2010). Una reflexión sobre los medios ciudadanos: esferas públicas, movilizadores de identidades y contra identidades de los sujetos políticos en Colombia. Encuentros 15 (2), 3344. Recuperado de http://repositorio.uac.edu.co/handle/11619/1184 
Ossa, C., Palma, M., Lagos, N., Quintana, I, Diaz, C (2017). Análisis de instrumentos de medición del pensamiento crítico. Ciencias Psicológicas 11 (1), 19 - 28. Recuperado de http://www. scielo.edu.uy/pdf/cp/v11n1/1688-4221-cp-11-01-00019.pdf

Oliveras, B \& Sanmartí N. (2009). La lectura como medio para desarrollar el pensamiento crítico en $8^{\mathrm{a}}$ convención nacional y $1^{\mathrm{a}}$ internacional de profesores de ciencias naturales. Recuperado de http://www.scielo.org.mx/pdf/eq/v20s1/v20s1a5.pdf

Organización de las Naciones Unidas para la Educación, la Ciencia y la Cultura. (2013). Enfoques estratégicos sobre las TIC en educación en América latina y el caribe 2013. Recuperado 4 de diciembre de 2016 de http://www.unesco.org/new/fileadmin/MULTIMEDIA/FIELD/Santiago/ images/ticsesp.pdf

Paul, R., \& Elder, L. (2005). Estándares de competencia para el pensamiento crítico. Estándares, Principios, Desempeño, Indicadores y Resultados. Con una Rúbrica Maestra en el Pensamiento Crítico. Fundación para el pensamiento crítico

Ortiz, E (2015). Actividades y tareas de Escritura Académica en el Contexto Universitario (pregrado) Ikala, revista de lenguaje y cultura. 20 (3), 343- 358. Recuperado de https://www.redalyc.org/ pdf/2550/255042795005.pdf

Sánchez, J., Brito, N. (2015). Desarrollo de competencias comunicativas, mediante la lectura crítica, escritura creativa y expresión oral. Revista Encuentros, Universidad Autónoma del Caribe, 13 (1), 117-141. Recuperado de http://repositorio.uac.edu.co/handle/11619/2083?show=full

Saiz, C \& Fernández, S. (2012). Pensamiento crítico y aprendizaje basado en problemas cotidianos. Revista de Docencia Universitaria 10 (3), 325 - 346. Recuperado de http://red-u.net/redu/ files/journals/1/articles/296/public/296-1668-1-PB.pdf

Zemelman, H. Pensar teórico y pensar epistémico (2004). Los retos de las ciencias sociales latinoamericanas. Instituto Pensamiento y cultura en América Latina, México.

\section{Para citar este artículo}

Santacoloma, L. (2020). Agro-Relatos. Periódico Universitario para el desarrollo de competencias de escritura y pensamiento crítico en estudiantes de Ciencias Agrarias. Revista Luciérnaga Comunicación. Vol.12 Núm. 23. Pp:177-192. https://doi.org/10.33571/revistaluciernaga.v12n23a9

OJS: https://revistas.elpoli.edu.co/index.php/luc/issue/archive

Link: https://www.politecnicojic.edu.co/index.php/revista-luciernaga 